\title{
Validação do modelo LES PALM por meio de dados de radiosondagens e de aeronave coletados durante o Experimento GoAmazon
}

\author{
Validation of PALM LES model by using radiosounding and aircraft data from \\ GoAmazon field campaigns
}

Thomas Kaufmann e Gilberto Fisch

Instituto Nacional de Pesquisas Espaciais, Brasil

tsskfmn@gmail.com; fisch.gilberto@gmail.com

\begin{abstract}
Resumo
A evolução da camada limite convectiva sobre superfície heterogênea simulada pelo modelo LES PALM é comparada com dados de radiosondagem e de aeronave obtidos do Experimento GoAmazon, realizado durante as estações chuvosa e seca de 2014 na Floresta Amazônica. É mostrado que, de uma forma geral, o crescimento da camada limite convectiva foi bem representado pelo modelo PALM durante os dois casos se comparados as observações. O modelo tende a subestimar a espessura da camada limite convectiva durante o período da manhã. Por outro lado, responde satisfatoriamente ao decréscimo dos forçantes de superfície no final da tarde.
\end{abstract}

Palavras-chave: heterogeneidade superficial, camada limite convectiva, medições por aeronave, modelo LES PALM, GoAmazon.

\begin{abstract}
In the present study, the evolution of the convective boundary layer over heterogeneous surface simulated by PALM LES is validated with radiosounding and airborne data from GoAmazon field campaigns, held in Amazon Rainforest during the 2014 wet and dry seasons. It is shown that, in general case, the growth of the convective boundary layer simulated by PALM compares well with observational data. However, during the morning time, the convective boundary layer depth is underestimated, whereas it showed acceptable response to the decreasing of the surface forcings along the late afternoon.
\end{abstract}

Keywords: surface heterogeneity, convective boundary layer, airborne measurements, PALM LES model, GoAmazon. 


\section{Introdução}

O Experimento GoAmazon (Green Ocean Amazon) ocorrido na Amazônia entre Fevereiro e Outubro de 2014 teve como objetivo aferir a interação entre a pluma de poluição gerada na cidade de Manaus/Amazonas, as nuvens e a floresta. Uma rede de estações coletou dados em superfície (medições convencionais, fluxos, entre outros), realizou lançamento de radiosondagens e detecção de células convectivas através de um radar; enquanto que simultaneamente uma aeronave de pesquisa - Gulfstream-1 (Schmid et al., 2014) - amostrou características da camada limite planetária (CLP) e a atmosfera livre.

$\mathrm{O}$ uso da aeronave permitiu obter informações de fluxos turbulentos dentro da camada de nuvens, no topo e fora da camada limite planetária - regiões da troposfera em que torres micrometeorológicas não cobrem (LeMone e Pennell, 1980). Os dados geralmente utilizados para cálculo de fluxos são aqueles medidos em legs, que são trechos em que a aeronave sobrevoa a uma altura praticamente constante, permitindo ter o mínimo de influência de mudanças abruptas de nível de voo (Lenschow, 1970). O objetivo principal deste trabalho é comparar os fluxos de calor sensível obtidos a partir da aeronave com aqueles gerados por um modelo de simulação de grandes vórtices chamado PALM - Parallelized Large-eddy simulation Model (Raasch e Schroter, 2001) - sobre a área do T3 (sítio localizado em região de heterogeneidade superficial em Manaus). Também são feitas comparações entre os perfis verticais de temperatura potencial obtidos por radiosondagem e pelo modelo PALM, a fim de validar qualitativamente o desempenho do PALM ao simular a evolução do crescimento da camada limite convectiva (diurna) sobre heterogeneidade superficial durante as estações chuvosa e seca.

\section{Metodologia}

No cálculo do fluxo de calor sensível, foram utilizadas medidas de velocidade vertical $w$ e temperatura potencial $\theta$ obtidas em alta frequência $(20 \mathrm{~Hz})$ a partir de um voo da aeronave durante a estação chuvosa (13/03/2014) e outro durante a estação seca (13/09/2014) sobre a área de heterogeneidade superficial em torno do sítio T3 (localização: $\left.3^{\circ} 12^{\prime} 36.0^{\prime \prime} \mathrm{S}, 60^{\circ} 36^{\prime} 00.0^{\prime \prime} \mathrm{W}\right)$.

A região possui áreas de floresta com diversos trechos desmatados (Figura 1). O fetch máximo em torno do sítio é de aproximadamente $8,9 \mathrm{~km} \times 6,9 \mathrm{~km}$, sendo que a maior parte da região heterogênea está situada a jusante do escoamento médio cuja direção climatológica

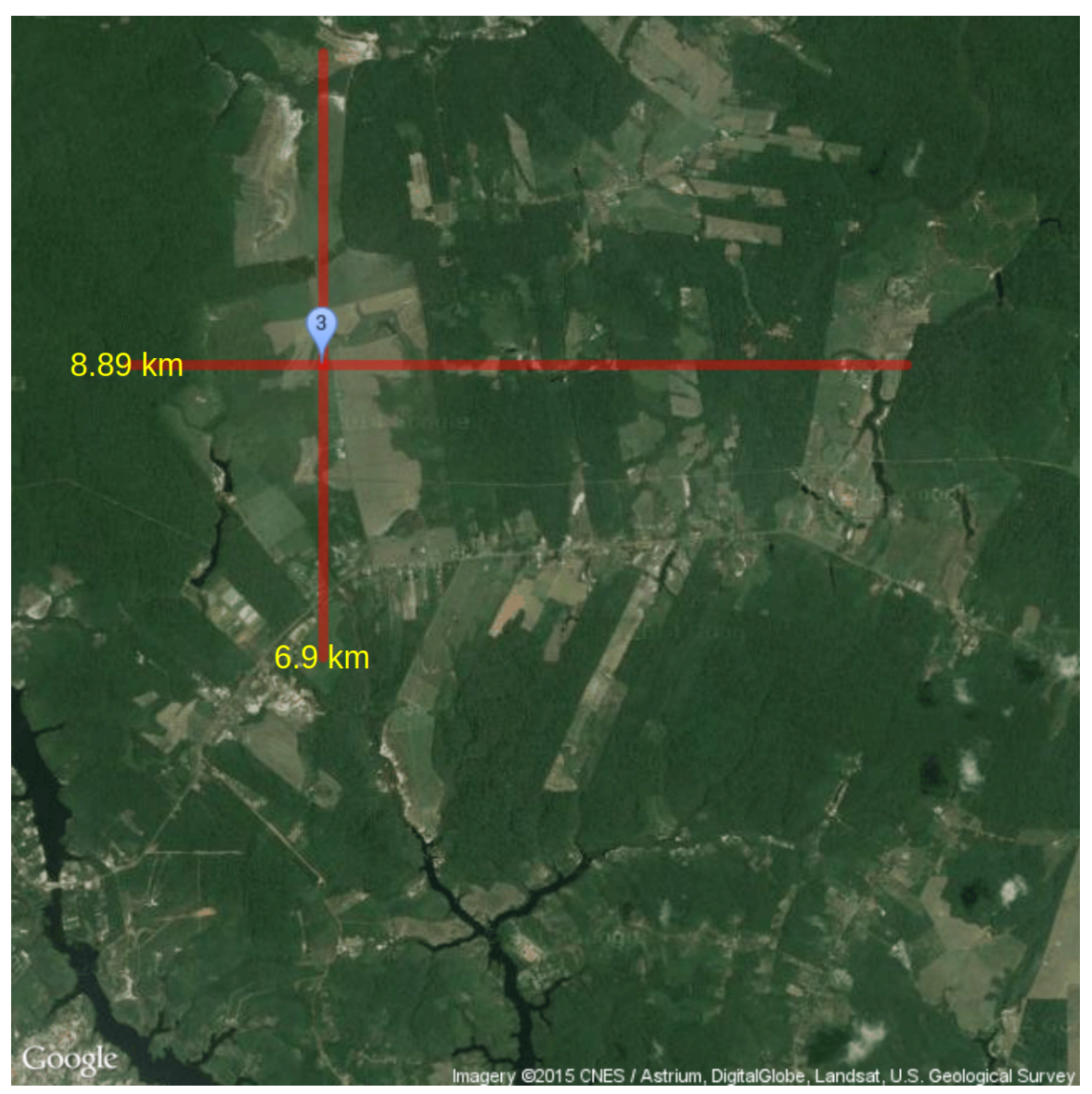

Figura 1: Localização aproximada do sítio T3 (marcador azul), mostrando a área representativa (fetch) das medições por instrumentos em superfície, por radiosondagem e por aeronave. (Adaptado de: Google Inc.) 
predominante na baixa troposfera é de leste/nordeste. Algumas informações de cada leg são mostradas na Tabela 1, tais como horários e altura de voo, bem como as características da CLP naquele nível (observadas a partir dos perfis de radiosondagem). Todos os tempos são considerados em horário local.

O tratamento preliminar das séries de $w$ e $\theta$ consistiu na aplicação da média de Reynolds, afim de obter as flutuações $w^{\prime}$ e $\theta^{\prime}$ (Lenschow, 1970); e na posterior remoção de eventuais tendências lineares ou polinomiais, de modo a eliminar do sinal da leg a influência de processos de maior escala (Lenschow et al., 1994; Mahrt, 1997; Vickers e Mahrt, 1997).

Conforme sugerido por Mallat (1989), Lenschow et al. (1994), Howell e Mahrt (1997) e Vickers e Mahrt (2003), cada leg foi particionada em grupos de janelas de aproximadamente $0,25 \mathrm{~km} ; 0,5 \mathrm{~km} ; 1 \mathrm{~km} ; 2 \mathrm{~km} ; 4 \mathrm{~km} ; 8 \mathrm{~km}$ e, quando possível, $15 \mathrm{~km}$.

A estacionariedade de segunda ordem da covariância foi testada em cada grupo de janelas - isto é, primeiramente nas janelas de $0,25 \mathrm{~km}$, depois nas janelas de $0,5 \mathrm{~km}$, e assim consecutivamente - a partir do método desenvolvido por Nason (2013).

Os grupos de janelas de $0,25 \mathrm{~km}$ a $2 \mathrm{~km}$ foram os que garantiram $100 \%$ de estacionariedade de segunda ordem. Assim, o particionamento em janelas de $2 \mathrm{~km}$ foi escolhido para o cálculo dos fluxos, devido a capacidade de capturar um número máximo de vórtices na camada limite convectiva sem perder a estacionariedade do sinal. A estacionariedade é um fator fundamental para a ergodicidade do sinal, que por sua vez é a premissa que garante a validade do método da covariância de vórtices utilizado no presente estudo (Aubinet et al., 2012).

O fluxo de calor sensível foi integrado em cada grupo de janelas conforme a (Equação 1):

$$
H=\int_{t_{0}}^{t} \overline{w^{\prime} \theta^{\prime}} d t
$$

em que $\overline{w^{\prime} \theta^{\prime}}$ denota a média da covariância entre as flutuações da velocidade vertical e da temperatura potencial, enquanto $t_{0}$ e $t$ são os tempos inicial e final da janela, respectivamente. O fluxo $H$ nesse caso é apresentado aqui em termos de unidade cinemática $(\mathrm{K} . \mathrm{m} / \mathrm{s})$; no entanto, é possível transformá-lo para unidades de energia $\left(\mathrm{W} / \mathrm{m}^{2}\right)$ multiplicando pelo fator $\rho c_{p}$.

Obteve-se um valor representativo de fluxo de calor sensível para a região do sítio aplicando a média aritmética das janelas cuja latitude/longitude coincidiram com a área de heterogeneidade superficial (fetch) em T3.

A evolução da camada limite convectiva de dois dias selecionados como representativos dos períodos chuvoso (13/03/2014, sim_20140313) e seco (13/09/2014, sim_20140913) foi simulada utilizando o modelo tridimensional PALM com as seguintes configurações:

A grade possui $(x, y, z)=(200,200,80)$ pontos, com distanciamento de $50 \mathrm{~m}$ entre os pontos nas três dimensões. O que resulta em um domínio de $(x, y, z)=(10,10,4)$ $\mathrm{km}$.

O tempo de integração do modelo foi de 13 horas (compreendendo o período das 08:00 as 21:00 horário local). O tempo de spin-up das duas simulações foi de aproximadamente 3600 segundos (1 hora), isto é, esse é o tempo que o modelo leva para criar os primeiros vórtices turbulentos e atingir o equilíbrio estatístico dadas as condições iniciais.

Fluxos de calor sensível (Figuras $3 b$ e $5 b$ ) e de calor latente observados sobre a área de T3 foram inseridos como forçantes de superfície a cada 30 minutos. Foi utilizado o comprimento de rugosidade de $0,02 \mathrm{~m}$ estabelecido pela Agência USGS (United States Geological Survey) para pastagem em área de heterogeneidade superficial.

Tabela 1: Altura e região da camada limite planetária correspondente a cada leg dos voos selecionados sobre T3.

13/03/2014

\begin{tabular}{llr}
\hline Leg (Horário) & Altura $(\mathrm{m})$ & Características da CLP \\
\hline leg09 (11:05:56-11:13:52) & $413,45 \pm 7,81$ & $\begin{array}{r}\text { camada de mistura } \\
\text { camada de mistura }\end{array}$ \\
leg13 (11:39:29-11:53:26) & $411,58 \pm 3,73$ & \\
leg21 (12:37:40-12:45:05) & $1034,17 \pm 9,27$ & zona de entranhamento \\
\hline & $\mathbf{1 3 / 0 9 / 2 0 1 4}$ & \\
\hline Leg (Horário) & Altura $(\mathrm{m})$ & Características da CLP \\
\hline leg06 (11:22:45-11:26:52) & $447,35 \pm 11,68$ & camada de mistura \\
leg11 (11:54:30-12:06:39) & $450,21 \pm 10,32$ & camada de mistura \\
leg13 (12:29:54-12:34:01) & $2069,29 \pm 11,01$ & atmosfera livre \\
leg16 (12:47:45-13:00:50) & $1414,57 \pm 13,71$ & zona de entranhamento \\
leg17 (13:04:14-13:18:27) & $756,17 \pm 14,36$ & camada de mistura \\
\hline
\end{tabular}


Os gradientes verticais de $\theta$, umidade específica $q$, componentes $u$ e $v$ do vento dentro da camada limite planetária foram obtidos a partir de dados de radiosondagens. Como o interesse é o de simular a camada limite convectiva (diurna), os perfis iniciais correspondem a primeira sondagem após o horário em que o fluxo de calor sensível em superfície se tornou positivo (geralmente, a radiosondagem das 8:00 horário local). Os valores simulados de fluxos de calor sensível que forem mencionados adiante referem-se ao fluxo total (integrado).

\section{Resultados e Discussões}

\subsection{Caso 1: sim_20140313 - estação chuvosa}

De modo geral, o modelo teve bom desempenho na simulação do desenvolvimento da camada limite convectiva quando comparado as observações por radiosondagem (Figura 2a). O mesmo pode ser afirmado em relação aos fluxos de calor sensível (Figura $2 b$ ).

Exceto no horário das 11:00, o perfil simulado de $\theta$ (Figura 2a) mostrou uma camada limite convectiva menos espessa do que a observação. Isto é, enquanto a sondagem indicava o topo entre 900-1000 m de altura, o PALM mostrou o topo entre 400 e $500 \mathrm{~m}$.

Ao passo que em torno de 14:00, horário após o máximo de insolação e o máximo positivo do fluxo de calor sensível em superfície (Figuras 3a e 3b, respectivamente), a espessura da camada limite simulada foi semelhante a observada pela sondagem. Esse semelhança no período da tarde, de uma forma geral, concorda com os resultados de comparações obtidos por Maronga e Raasch (2012) para o mesmo período do dia, embora sob condições distintas da Amazônia e também muito mais complexas de heterogeneidade superficial.

A diferença entre o fluxo de calor sensível observado e o simulado às 13:00 (Figura $2 b$ ) pode estar associado $a$ trocas de calor mais intensas observadas àquela altura, uma vez que na referida leg a aeronave cruzou uma camada de nuvens. Evidentemente, o modelo não pôde capturar essa característica, uma vez que os parâmetros microfísica de nuvens e precipitação do modelo PALM não estavam ativados no momento da simulação.

Após as 16:00, período em que a insolação decresce (Figura 3a), é notável a sensibilidade do modelo ao decaimento dos fluxos de calor à superfície (Figura 3b), que é percebido através da diminuição da intensidade do gradiente vertical de $\theta$ e de $H$ (Figuras 2a e 2b, respectivamente) após aquele horário.

\subsection{Caso 2: sim_20140913 - estação seca}

Assim como no caso 1, o desenvolvimento da camada limite convectiva foi satisfatoriamente bem representado pelo modelo PALM, quando comparado as observações por radiosondagem (Figuras 4a e 4b). No entanto, em contraponto à simulação da estação chuvosa, o perfil simulado de $\theta$ (Figura 4a) em torno das 11:00 mostrou uma camada limite convectiva tão desenvolvida e profunda quanto a da observação. Algumas diferenças acima de $500 \mathrm{~m}$ são percebidas nesse mesmo perfil, possivelmente associadas a alguma dificuldade do modelo em representar a destruição da camada residual ao longo da manhã. Após as 14:00, horário imediatamente após o máximo de insolação e após o máximo positivo do fluxo de calor sensível em superfície (Figuras $5 a$ e $5 b$, respectivamente), a espessura da camada limite convectiva simulada foi bem semelhante a da observação, como mostrado na Figura 4a, o que novamente concorda com as comparações de Maronga e Raasch (2012). No que diz respeito aos perfis de fluxo de calor sensível, a diferença mais nítida entre simulado e observado pode ser notada as 12:00 em torno de $2070 \mathrm{~m}$ de altura (Figura $4 \mathrm{~b}$ ). Uma análise de covariância entre $w^{\prime}, \theta^{\prime}$ e $q^{\prime}$ mostrou que na referida leg, a aeronave percorreu uma camada sem nuvens cruzando com uma sequência de downdrafts (subsidência de ar quente e seco) na atmosfera livre. Esse fato fornece evidências para explicar grande parte da diferença entre o fluxo de calor sensível simulado e observado àquela altura. Maronga e Raasch (2012) também observaram essa limitação do PALM em relação a circulações secundárias em suas simulações sobre regiões de heterogeneidade superficial.

\subsection{Diferenças entre o caso chuvoso e o caso seco}

Não foram observadas diferenças significativas entre os casos simulados em relação a altura máxima da camada limite convectiva. No entanto, o crescimento na parte da manhã foi mais rápido no caso chuvoso do que no caso seco (Figuras 2a e 4a). A explicação pode estar nos valores menores de fluxo de calor sensível à superfície fornecidos antes do meio-dia na simulação do caso seco (Figura 5b). Por exemplo, em torno de 10:00 e 11:00, houve uma queda no valor do fluxo de $H$ à superfície e isso pode ter significado um retardo no crescimento da camada limite convectiva ao longo do final da manhã. 

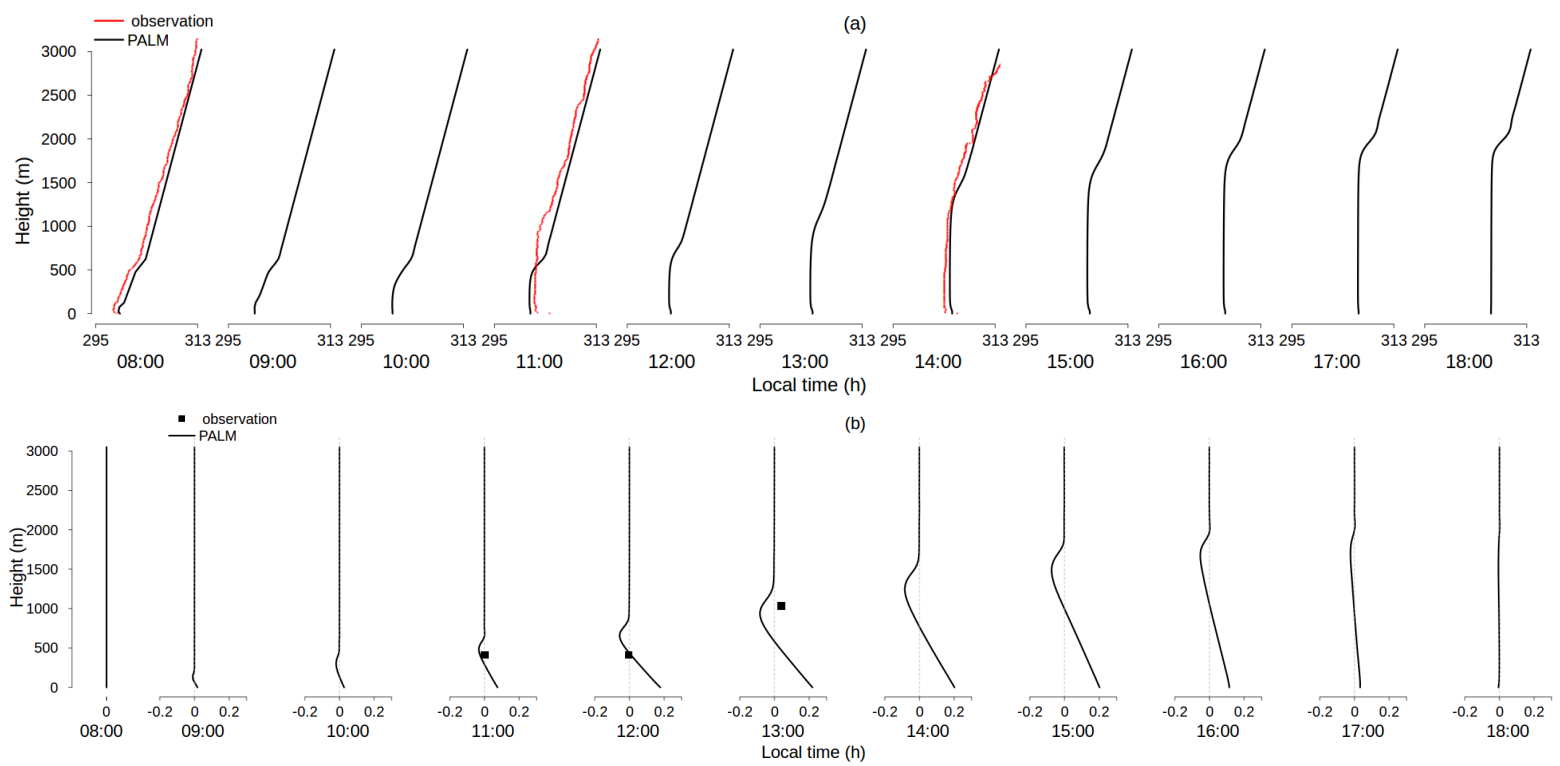

Figura 2: (a) Evolução temporal de perfis de temperatura potencial simulados (linha preta, em $K$ ) e observados (linha vermelha, em $K$ ) no T3 ao longo da manhã e tarde do dia 13/03/2014. (b) Evolução temporal dos perfis de fluxo de calor sensível simulados (linha preta, em $K . \mathrm{m} / \mathrm{s}$ ) e observações realizadas por aeronave (pontos pretos, em K.m/s) no mesmo período que em (a).
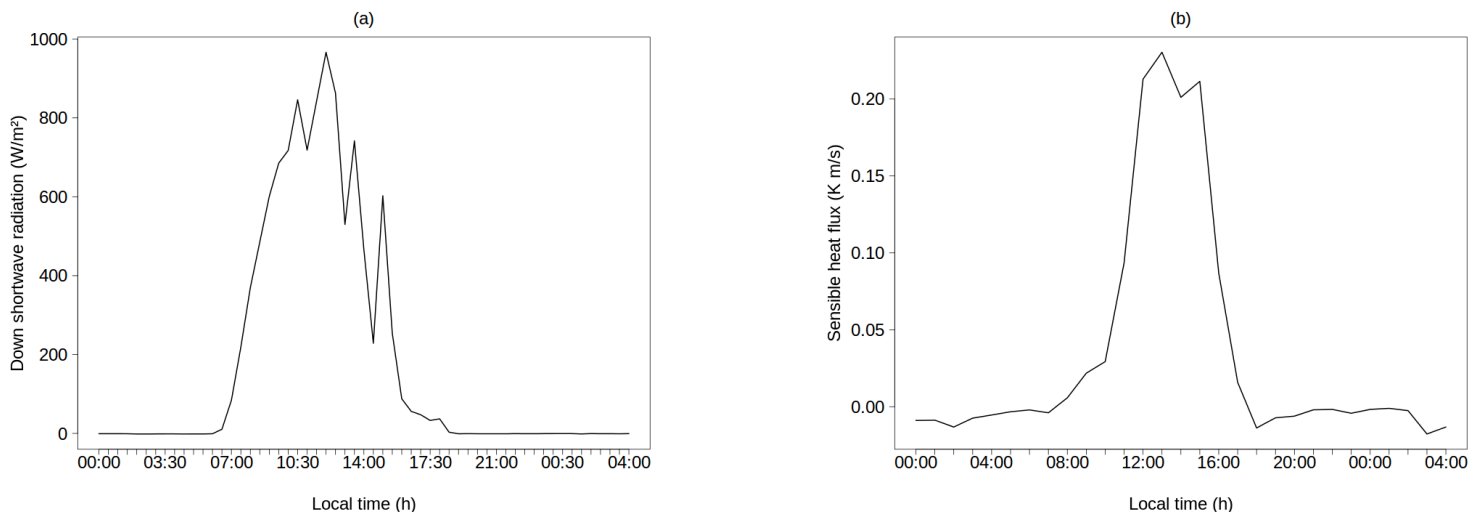

Figura 3: (a) Ciclo diário da radiação de onda curta incidente à superfície (médias de 30 min) e do (b) fluxo de calor sensível à superfície (médias de 60 min) no sítio T3 em 13/03/2014. 

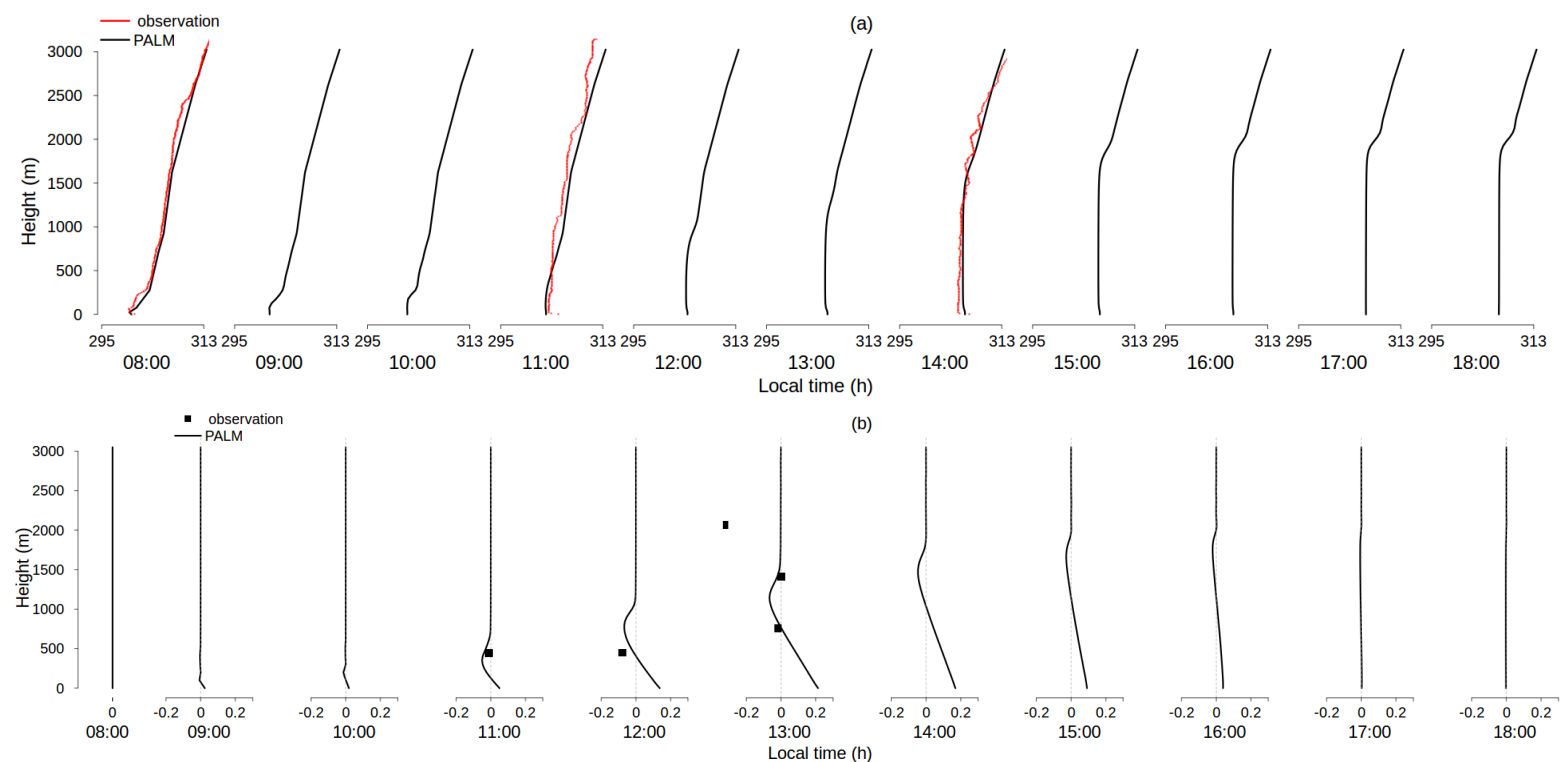

Figura 4: (a) Evolução temporal de perfis de temperatura potencial simulados (linha preta, em K) e observados (linha vermelha, em K) no T3 ao longo da manhã e tarde do dia 13/09/2014. (b) Evolução temporal dos perfis de fluxo de calor sensível simulados (linha preta, em K.m/s) e observações realizadas por aeronave (pontos pretos, em K.m/s) no mesmo período que em (a).
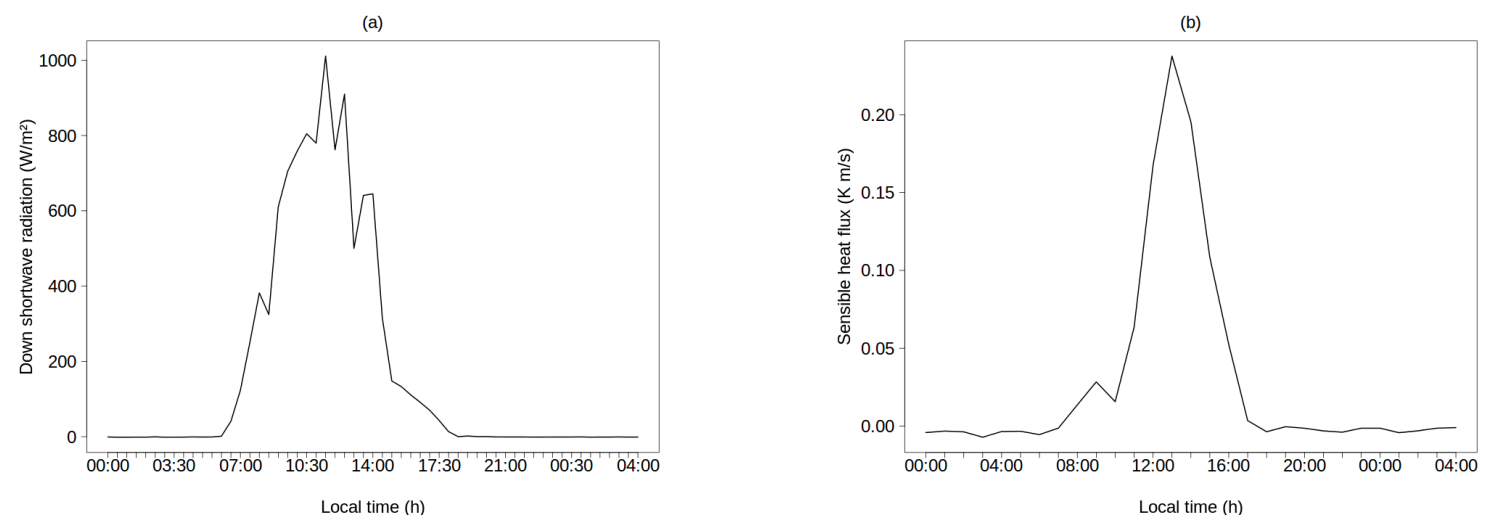

Figura 5: (a) Ciclo diário da radiação de onda curta incidente à superfície (médias de $30 \mathrm{~min}$ ) e do (b) fluxo de calor sensível à superfície (médias de $60 \mathrm{~min}$ ) no sítio T3 em 13/09/2014. 


\section{Conclusões}

De uma forma geral, o modelo PALM representou satisfatoriamente os perfis de temperatura potencial e de fluxo de calor sensível, porém, teve certa dificuldade em simular o crescimento da camada limite convectiva no período da manhã durante ambos os casos 1 (estação chuvosa) e 2 (estação seca). Outra limitação está associada a representação de circulações secundárias (downdrafts, por exemplo). Observou-se uma boa sensibilidade do modelo PALM ao decréscimo dos forçantes de superfície (fluxos de calor sensível) do meio da tarde até $\mathrm{o}$ anoitecer.

\section{Referências}

Aubinet, M., Vesala, T., Papale, D. (2012). Eddy-covariance: A practical guide to measurement and data analysis. Springer.

Howell, J. F., Mahrt, L. (1997). Multiresolution flux decomposition. Boundary-Layer Meteorology, 83(1), 117137.

LeMone, M. A., Pennell, W. T. (1980). A comparison of turbulence measurements from aircraft. Journal of Applied Meteorology, 19(1), 1420-1437.

Lenschow, D. (1970). Airplane measurements of planetary boundary-layer structure. Journal of Applied Meteorology, 9(1), 874-884.

Lenschow, D. H., Mann, J., Kristensen, L. (1994). How long is long enough when measuring fluxes and other turbulence statistics? Journal of Atmospheric and Oceanic Technology, 11(1), 661-673.

Mahrt, L. (1997). Flux sampling errors for aircraft and towers. Journal of Atmospheric and Oceanic Technology, 15(1), 416-429.

Mallat, S. G. (1989). A theory of multiresolution signal decomposition: The wavelet representation. IEEE Transactions on Pattern Analysis and Machine Intelligence, 11(1), 674-693.

Maronga, B., Raasch, S. (2012). Large-eddy simulations of surface heterogeneity effects on the convective boundary layer during the litfass-2003 experiment. Boundary-Layer Meteorology, 146(1), 17-44.

Nason, G. (2013). A test for second-order stationarity and approximate confidence intervals for localized autovariances for locally stationary timeseries. Journal of the Royal Statistical Society: Series B - Statistical Methodology, 75(5), 879-904.
Raasch, S., Schroter, M. (2001). Palm - a large-eddy simulation model performing on massively parallel computers. Meteorologische Zeitschrift, 10(5), 363-372.

Schmid, B., Tomlinson, J. M., Hubbe, J. M., Comstock, J. M., Mei, F., Chand, D., Pekour, M. S., Kluzek, C. D., Andrews, E., Biraud, S. C., McFarquhar, G. M. (2014). The doe arm aerial facility. Bulletin of American Meteorological Society, 95(5), 723-742.

Vickers, D., Mahrt, L. (1997). Quality control and flux sampling problems for tower and aircraft data. Journal of Atmospheric and Oceanic Technology, 14(1), 512-526.

Vickers, D., Mahrt, L. (2003). The cospectral gap and turbulent flux calculations. Journal of Atmospheric and Oceanic Technology, 20(1), 660-672. 